\title{
Sporanox en la candidiasis vaginal
}

\author{
Dres. José A. Daunas*, Alvaro Orlando Sarmiento*
}

RESUMEN. Pacientes con candidiasis vaginal fueron seleccionadas a participar en un estudio multicéntrico abierto para evaluar el SPORANOX (ITACONAZOL Janssen) un día de tratamiento (dos cápsulas 100 mg tomadas en la mañana y en la noche del mismo día) para evaluar el flujo y la sintomatología vulvar y vaginal (eritema, edema, prurito); se realizó test de KOH y cultivo a la admisión, una semana después de tratamiento y a las cuatro semanas. Los resultados indican una mejoría significativa de la sintomatología del flujo en la primera semana de observación y $96.5 \%$ en la última semana. Los test del laboratorio y cultivo indican que 311 de 368 pacientes fueron negativas en el $85 \%$ después de la primera semana. Al final de la observación clínica hecha en 266 pacientes incluían cultivos de control de los cuales 240 fueron negativos, indicando una rata de curación del 92\%. No se detectaron efectos colaterales. (Rev Col Obstet Ginecol 1991; 42(3): 219-221).

PALABRAS CLAVES: Estudio multicéntrico abierto, candidiasis vaginal.

SUMMARY. Patients with vaginal candidiasis were selected to participate in a multicentric open study to evaluate the one day treatment with SPORANOX (ITRACONAZOLE Janssen) (two capsules $100 \mathrm{mg}$ take morning and evening of the same day) laboratory test $(\mathrm{KOH})$ and culture as well evaluation of the leucorrhea and vulvar and vaginal symptomatology (erythema, oedema, soreness and pruritus) were perform at the admision, one week after treatment and finally 4 weeks after. The results indicated a significative improvement of the symptomatology as well in leucorrhea, at the first week of observation to continue to reach a $96.5 \%$ at the last observation. Laboratory test and culture indicated that 311 out of 368 patients became negative $85 \%$ after the first week. By the end of the clinical observation done in 262 patients including the positive ones left from previous control 240 were negative indicating a $92 \%$ of cure rate. No side effects related to the treatment were reported the positive ones left from.

KEY WORDS: Multicentric open study, vaginal candidiasis.

\section{Introducción}

Es bien conocida la incidencia que dentro de todas las vaginitis tiene la infección micótica producida por la candida spp. Se ha estimado que el $75 \%$ de las mujeres presentan por lo menos un episodio de candidiasis vaginal durante sus años reproductivos (1). Otras causas de vaginitis como las bacterias y las trichomonas vaginalis fueron y son tratadas con medicaciones orales; pero la candidiasis vaginal sólo lo fue recientemente con el advenimiento del Ketoconazol, el primer anti-micótico de uso oral (2). Si bien algunos derivados imidazólicos han demostrado poseer acción, tanto sobre las formas de levadura, como sobre las hifas invasivas de la candida (3), uno de los últimos en obtenerse, el Itraconazol (Sporanox-Janssen), ha demostrado además, debido a sus características clínico-farmacológicas, ser el ideal de los antimicóticos, dada su concentración y permanencia en la mucosa vaginal con concentraciones que sobrepasan las de los niveles sanguíneos, gracias a su afinidad por los tejidos después de su administración sistémica $(4,5,6,7)$.

Habiéndose comprobado la utilidad del Itraconazol (Sporanox de Janssen) en el tratamiento de la candidiasis vaginal durante un solo día de tratamiento (8) y conociéndose en nuestro medio un estudio con un menor número de pacientes

\footnotetext{
* Departamento de Medicina de los hospitales de la Samaritana y San Blas de Bogotá, respectivamente. Miembros del Janssen Research Council-Colombia. A.A. 047303.
}

(9), fue de nuestro interés y de muchos médicos del país el estudiar en un adecuado número de pacientes el valor de Sporanox en el tratamiento de la candidiasis vaginal, dentro de las pautas usualmente seguidas en la práctica médica y condiciones de nuestro medio.

\section{Pacientes y métodos}

a) Protocolo

Se desarrolló un protocolo que incluía: 1) Aspectos de ingreso al estudio: el presentar vaginitis de tipo crónica o aguda; 2) Valoración de la leucorrea y los síntomas vulvares y vaginales antes, a la semana y al mes del tratamiento; 3 ) El soporte de diagnóstico basado en el cultivo, el frotis y (KOH) para evidenciar la presencia de candida albicans; 4) La edad de las pacientes requería ser de más de 14 años, y 5) Las causas de rechazo para no incluirse en el estudio fueron existencia de embarazo o vaginitis de otras causas o agregadas a la candidiasis.

Se solicitó información sobre antecedentes de tratamientos previos y existencia o no de terapia anticonceptiva en el momento de inclusión al estudio. Lógicamente se señaló un tiempo mínimo de una semana de duración con relación a cualquier terapia previa antimicótica antes de iniciarse el tratamiento.

b) Tipo de estudio

Se trató de un estudio multicéntrico abierto controlado médicamente y por laboratorio a la inclusión y a las semanas 
1a. y 4a. después del tratamiento.

c) Tratamiento-Droga

Cada paciente debía ser tratada con cuatro (4) cápsulas de Itraconazol (Sporanox) de 100 mgs para tomar dos cápsulas en la mañana y dos en la tarde del mismo día. Se recomendó realizarse la toma con las comidas.

d) Evaluación de resultados

Además de valorar los cambios sobre los síntomas vulvares y vaginales, cada observador debió de realizar su evaluación indicando curación (sí o no) y soportando la misma con cultivo (positivo o negativo) o bien $\mathrm{KOH}$ (positivo o negativo). Los informes del estudio fueron analizados y estadísticamente valorados por Luis Angel Rodríguez, estadístico de la U.N., y por SIMES (sistemas y métodos estadísticos)

\section{Resultados}

Se incluyeron para el estudio final 493 pacientes de un total de 501, que siguieron las normas indicadas de inclusión. Del total de 493 el $62 \%$ o sea 308 seguían terapia anticonceptiva al momento de recibir tratamiento para la vaginitis.

- Centros participantes

Ciento veintidós (122) médicos de las principales ciudades del país participaron en el tratamiento de las 493 pacientes. Según su especialidad fueron $51 \%$ ginecólogos, $29 \%$ médicos generales y $20 \%$ especialistas de medicina interna o familiar.

- Aspectos de admisión

a) Edad: Las pacientes incluidas en el estudio tenían de 15 a 65 años, así: $24 \%$ de los 15 a los 29 años (43\%) o sea 119 pacientes; 215 de los 25 a los 34 años; 112 de los 35 a los 44 años (22\%); el resto fueron mayores de 45 años (9\%). El promedio fue de 30 años de edad con peso promedio de $56 \mathrm{~kg}$ y talla media de $1.60 \mathrm{mts}$ de estatura.

Cuadro 1

EVALUACION CLINICA DEL TRATAMIENTO AL INICIO VS. UN MES EN LEUCORREA

\begin{tabular}{|c|c|c|c|c|c|c|c|c|c|c|}
\hline \multirow{3}{*}{$\begin{array}{l}\text { Evaluación } \\
\text { al Inicio }\end{array}$} & \multicolumn{2}{|c|}{ Total } & \multicolumn{8}{|c|}{ Evaluación al Mes del Tratamiento } \\
\hline & \multirow[t]{2}{*}{$\mathbf{N}$} & \multirow[t]{2}{*}{$\%$} & \multicolumn{2}{|c|}{ Ausente } & \multicolumn{2}{|c|}{ Ligero } & \multicolumn{2}{|c|}{ Moderado } & \multicolumn{2}{|c|}{ Severo } \\
\hline & & & $\mathbf{N}$ & $\%$ & $\mathbf{N}$ & $\%$ & $\mathbf{N}$ & $\%$ & $\mathbf{N}$ & $\%$ \\
\hline Ausente & 6 & 2.3 & 6 & 2.3 & & & & & & \\
\hline Ligero & 53 & 20.1 & 43 & 16.3 & 9 & 3.4 & 1 & .4 & & \\
\hline Moderado & 118 & 44.7 & 89 & 33.7 & 22 & 8.3 & 6 & 2.3 & 1 & .4 \\
\hline Severo & 87 & 33.0 & 59 & 22.3 & 24 & 9.1 & 3 & 1.1 & 1 & .4 \\
\hline TOTAL & 264 & 100 & 197 & 74.6 & 55 & 20.8 & 10 & 3.8 & 2 & .8 \\
\hline
\end{tabular}

SIMES-Sistemas y Métodos Estadísticos.

Cuadro 2

EVALUACION CLINICA DEL TRATAMIENTO AL INICIO VS. PRIMERA SEMANA EN LEUCORREA

\begin{tabular}{|c|c|c|c|c|c|c|c|c|c|c|}
\hline \multirow{3}{*}{$\begin{array}{c}\text { Evaluación } \\
\text { al Inicio }\end{array}$} & \multicolumn{2}{|c|}{ Total } & \multicolumn{8}{|c|}{ Evaluación al Mes del Tratamiento } \\
\hline & \multirow{2}{*}{$\mathbf{N}$} & \multirow{2}{*}{$\%$} & \multicolumn{2}{|c|}{ Ausente } & \multicolumn{2}{|c|}{ Ligero } & \multicolumn{2}{|c|}{ Moderado } & \multicolumn{2}{|c|}{ Severo } \\
\hline & & & $\mathbf{N}$ & $\%$ & $\mathbf{N}$ & $\%$ & $\mathbf{N}$ & $\%$ & $\mathbf{N}$ & $\%$ \\
\hline Ausente & 8 & 2.2 & 8 & 2.2 & & & & & & \\
\hline Ligero & 80 & 21.5 & 63 & 16.9 & 15 & 4.0 & 2 & .5 & & \\
\hline Moderado & 170 & 45.7 & 86 & 23.1 & 75 & 20.2 & 9 & 2.4 & & \\
\hline Severo & 114 & 30.6 & 36 & 9.7 & 63 & 16.9 & 14 & 3.8 & 1 & .3 \\
\hline TOTAL & 372 & 100 & 193 & 51.9 & 153 & 41.1 & 25 & 6.7 & 1 & .3 \\
\hline
\end{tabular}

SIMES-Sistemas y Métodos Estadísticos.
Cuadro 3

RESOLUCION DE SINTOMAS POR NUMERO DE PACIENTES

\begin{tabular}{|c|c|c|c|c|c|c|c|c|}
\hline \multirow{3}{*}{$\begin{array}{l}\begin{array}{l}\text { Sintomas } \\
\text { de las } \\
\text { Pacientes }\end{array} \\
\end{array}$} & \multirow{3}{*}{$\begin{array}{l}\text { Antes del } \\
\text { Tratamiento } \\
\text { No. de Pts. }\end{array}$} & \multicolumn{7}{|c|}{ Después del tratamiento ResoluciónDiferencias } \\
\hline & & AlaS & emant & a $\mathrm{All}$ & Mes & No. & $\%$ & Estadísticas \\
\hline & & Igual & Peor & Igual & Peor & Pts. & & $\mathrm{P} / 0.0001$ \\
\hline \multicolumn{9}{|c|}{ Vulvares: } \\
\hline Eritema & 285 & 17 & 3 & 4 & 1 & 280 & 98 & Significativa \\
\hline Edema & 198 & 8 & 1 & 4 & 0 & 194 & 97 & Significativa \\
\hline Dolor & 181 & 9 & 0 & 5 & 0 & 176 & 97 & Significativa \\
\hline Prurito & 332 & 18 & 2 & 12 & 3 & 317 & 95 & Significativa \\
\hline \multicolumn{9}{|l|}{ Vaginales: } \\
\hline Eritema & 281 & 21 & 3 & 8 & 2 & 271 & 96 & Significatıva \\
\hline Edema & 181 & 11 & 1 & 6 & 3 & 172 & 95 & Significativa \\
\hline Dolor & 180 & 6 & 1 & 3 & 0 & 177 & 98 & Significativa \\
\hline Prurito & 312 & 13 & 2 & 7 & 3 & 302 & 96 & Significativa \\
\hline
\end{tabular}

Cuadro 4

PACIENTES QUE CURARON, SEGUN EL INVESTIGADOR

\begin{tabular}{|c|c|c|c|c|}
\hline \multirow[t]{2}{*}{ Indicación de Curación } & \multicolumn{2}{|c|}{ A la Semana } & \multicolumn{2}{|c|}{ AlMes } \\
\hline & No. & $\%$ & No. & $\%$ \\
\hline Sí & 311 & 85 & 240 & 92 \\
\hline No & 57 & 15 & 22 & 8 \\
\hline TOTAL & 368 & 100 & 262 & 100 \\
\hline
\end{tabular}

b) Síntomas: Los síntomas más importantes en orden de frecuencia, por las cuales consultaron las pacientes, fueron: leucorrea $348(70 \%)$, prurito 72 (14\%), ardor vaginal; otros $25(5 \%)$; y otros variados o no mencionados $48(9 \%)$. Esa sintomatología correspondió a 295 pacientes de tipo agudo $(59 \%)$ y a 148 de tipo crónico $(30 \%)$. Las restantes fueron 50 pacientes no diferenciadas.

c) Tratamientos previos: Ciento cincuenta y una pacientes habían recibido tratamiento previo (151 o $30.6 \%$ de las pacientes). Los tratamientos se habían efectuado con una de las siguientes medicaciones: 45 Clotrimazol; 15 Ketoconazol; 14 Isoconazol; 11 Metronidazol; 10 Nistatina; 6 Talceclin, y 39 otros. El tiempo promedio de tratamiento con los mismos fue de una semana de duración. No obstante, 70 de esas pacientes $(41 \%)$ indicaron que emplearon una sola dosis en su tratamiento. Aparentemente en $95(63 \%)$ de ellas el tratamiento no produjo curación y $56(27 \%)$ informaron tratarse de reinfección su padecimiento actual.

d) Diagnóstico: El diagnóstico se evidenció por frotis positivo en el $70 \%$ de las pacientes y en el $27 \%$ por $\mathrm{KOH}$. Las restantes presentaron cultivo positivo para aceptación a la admisión al estudio.

\section{Tratamiento}

Todas las pacientes tomaron dos cápsulas de Sporanox $\times 100$ mgs en la mañana y dos en la tarde (cuatro al día) en un solo día de tratamiento. El Cuadro 1 muestra la evaluación que el síntoma leucorrea presentó en las 364 pacientes que lo presentaron. 152 mejoraron una esca':1; 149 dos escalas, y 36 tres escalas. Sólo 25 casos no presentaron mejoría y dos empeoraron. Al mes (Cuadro 2) mejoraron 68 en una escala; 113 en dos escalas, y 59 en tres escalas; 16 no presentaron mejoría y dos (2) empeoraron. Esos resultados son significativos estadísticamente.

En forma semejante se tabularon los resultados sobre los síntomas. Ellos se presentan resumidos en el Cuadro 3. Los resultados muestran que la sintomatología se redujo en su 
casi totalidad como lo indican las diferencias al final de la observación de cuatro (4) semanas (un mes). Cabe señalar cómo se evidencia que la mejoría obtenida a la semana se extiende hacia el final de la observación y llega a límites muy significativos, después de aplicarse el valor calculado de la estadística de Wilcoxon, la cual se aproxima a una distribución normal estandarizada Z-2, debido al número considerable de pacientes en estudio. Para una probabilidad $\mathrm{P} / 0.00001$ que permite llegar a la conclusión de efectividad del producto.

La valoración estadística es altamente significativa demostrando que Sporanox es muy efectivo no sólo en el tratamiento de la vaginitis candidiásica y leucorrea, sino también en los síntomas vulvares y vaginales.

Del total de 493 pacientes incluidas en el estudio, el médico observador no reportó ni firmó en el espacio de valoración de curación el reporte en 125 de los casos. Es por ello que la curación se valora en los 368 casos realmente concluidos al respecto, en los cuales el observador dio su concepto y lo refrendó con resultados de laboratorio. Esta observación se repite al mes, tiempo en el cual la valoración se realizó en sólo 262 pacientes.

Aparentemente el incumplimiento en la obtención de los exámenes de laboratorio, así como el no asistir con puntualidad a las citas, originan fallas que el médico no puede evitar. Tomándose las valoraciones finales completas como se presentan en el Cuadro 4 (valores igualmente significativos), la curación clínica y bacteriológica fue del $85 \%$ a la semana después del tratamiento y del $92 \%$ al mes del mismo.

No hubo reporte de efectos secundarios inducidos por la medicación de un día.

\section{Discusión}

No cabe duda que la valoración de un tratamiento en una entidad como la candidiasis vaginal debido a la re-infección así como a las recaídas, es muy difícil de realizarse en forma inobjetable. Se precisaría para ello un medio cerrado y dentro de un protocolo comparativo con droga activa y placebo. En nuestro medio falta aún mucho por realızarse en cuanto a la educación del enfermo y su participación completa dentro de la realización de un estudio. Así como para el médico, en cuanto a realizar la adecuada selección de los pacientes, que conlleve al cumplimiento de lo ofrecido después de una explicación somera y detallada del estudio y su objetivo. No obstante, a falta de ello, una de las compensaciones es el número amplio de pacientes. Debemos indicar que si bien los observadores realizaron el estudio dentro de los parámetros indicados, algunos se desviaron en cumplir todos los requisitos. Es por ello que faltaron anotaciones completas y esperadas a ser registradas, por lo cual algunas observaciones no se pudieron aceptar dentro del marco estadístico para inclusión en la valoración final.

Este estudio multicéntrico ha demostrado que la sintomatología y la causa etiológica fueron significativamente controladas por el tratamiento con Itraconazol (Sporanox) un día, como lo evidencia el análisis de SIMES. Es un hecho que la candidiasis vaginal constituye una patología frecuente y extendida en nuestro medio, la cuảl amerita el estudio de su solución en forma adecuada.

Nuestros resultados concuerdan con los de otros investigadores $(8,9,10,11)$ y permiten concluir que Itraconazol (Sporanox) administrado en dosis de $200 \mathrm{mgs}$ dos veces al día es efectivo para el tratamiento de la candidiasis vaginal y que representa una terapéutica a la cual las pacientes se adhieren fácilmente lográndose una terapia efectiva y fácil de seguir. Además, cabe señalar que la terapia de un solo día puede ser la pauta eficaz para el tratamiento intermitente con miras a minimizar las recaídas y re-infecciones de las pacientes que adquieran candidiasis vaginal (10).

\section{Conclusiones}

Este estudio permite concluir que Itraconazol (Sporanox Janssen) administrado en dosis de $200 \mathrm{mgs}$ dos veces al día, es un antimicótico altamente efectivo y útil en el tratamiento de la candidiasis vaginal y que la medicación de un solo día no indujo efectos indeseables, confirmando su seguridad.

NOTA: Como un reconocimiento publicamos la lista de médicos participantes del presente estudio en la página 234 de este número.

\section{BIBLIOGRAFIA}

1. Berg AO. Establishing the cause of genitourinary symptoms in women in a family practice. JAMA 1984; 251: 620-625.

2. Bisschop MPJM, Merkus JMWM, Scheijgrond H, et al. Treatment of vaginal candidiasis with Ketoconazole, a new orally active antimycotic. Eur J Obstet Gynecol Reprod Biol 1979; 9(4): 253.

3. Odds FC, et al. Effects of Imidazole and Triazole derivative antifungal Compunds on the Growth and Morphological Development of Candida Albicans Hyphoe. J of General Microbiology (1985, 13 p. 2581-2589).

4. Van Cutsen J, Van Gerven F, Janssen PAJ. Activity of orally, topically and parentally administered Itraconazole in the treatment of superficial and deep mycosis: animal models. Rev Infect Dis 1987; 9(1): S15-S32.

5. Espinel-Ingroff A, Shadomy S, Gebbart RJ. In vitro studies with 51211 (Itraconazole) Antimicrob. Agents, Chemoth 1984; 26(1): 5-9.

6. Heykants J, et al. The Pharmacokinetics of Itraconazole in animals and man: an overview in recent trends in the Discovery, development and Evaluation of Antifugal Agents, Ed. R.A. Fromtling, J.R. Prous Science Publishers, S.A. 1987; 233-249.
7. Tarosa E, Cauwenbergh G, Cilli P, et al. Itraconazole pharmacokinetics in the female genital tract: Plasma and tissue levels in patients undergoing hysterectomy after a single dose of $200 \mathrm{mgs}$ Itraconazole. Eur J Obstet Gynecol Reprod Biol 1986: 23: 85.

8. Cauwenbergh G. Itraconazole: The first orally active antifugal for single-day treatment of vaginal candidiasis. Current Therapeutic Research 1987; 41(2) February 1-5.

9. Bueno MM, Alvarez VMI. Tratamiento actual de la candidiasis vaginal. Presentado al XI Congreso Colombiano de Medicina Interna. Cali, Colombia. Octubre 11-15, 1990. A ser publicado.

10. Van Heusden AM. Markus JMWM. Chronic recurrent candidiasis: easy to treat difficult to cure. Results of intermittent treatment with a new oral antifungant. Eur $\mathbf{J}$ of Obst and Gyneco Reprod Biology 1990; 35: 75-83.

11. Blach B, Barnard PG, et al. Itraconazole in the treatment of acute vaginal candidiasis. S Afr Med J 1988; 73: 172-173 